\title{
Two-dimensional control of light with light on metasurfaces
}

\begin{abstract}
Maria Papaioannou ${ }^{1}$, Eric Plum ${ }^{1}$, João Valente ${ }^{1}$, Edward TF Rogers $^{1,2}$ and Nikolay I Zheludev ${ }^{1,3}$
The ability to control the wavefront of light is fundamental to focusing and redistribution of light, enabling many applications from imaging to spectroscopy. Wave interaction on highly nonlinear photorefractive materials is essentially the only established technology allowing the dynamic control of the wavefront of a light beam with another beam of light, but it is slow and requires large optical power. Here we report a proof-of-principle demonstration of a new technology for two-dimensional (2D) control of light with light based on the coherent interaction of optical beams on highly absorbing plasmonic metasurfaces. We illustrate this by performing 2D all-optical logical operations (AND, XOR and OR) and image processing. Our approach offers diffractionlimited resolution, potentially at arbitrarily-low intensity levels and with $100 \mathrm{THz}$ bandwidth, thus promising new applications in space-division multiplexing, adaptive optics, image correction, processing and recognition, 2D binary optical data processing and reconfigurable optical devices.
\end{abstract}

Light: Science \& Applications (2016) 5, e16070; doi:10.1038/lsa.2016.70; published online 22 April 2016

Keywords: all-optical data processing; all-optical image processing; coherent control; metasurfaces

\section{INTRODUCTION}

Virtually every optical system relies on control over light propagation by modifying the phase or intensity across a beam of light. Lenses, prisms, gratings and transformation optics devices ${ }^{1}$ such as optical cloaks $^{2-4}$ all redirect light by engineering the spatial distribution of optical constants in two (2D) or three dimensions (3D). Recently, several groups ${ }^{5-13}$ demonstrated that the propagation direction of light can also be controlled by phase-gradient metasurfaces, where spatially varying resonators on an interface scatter with different phases. Although this approach offers a powerful solution for static wavefront control, it does not offer dynamic control over light propagation. Established solutions for dynamic wavefront control use either electrooptical spatial light modulators based on liquid crystals or digital micromirror devices ${ }^{14-16}$, or all-optical methods based on holographic operations in nonlinear photorefractive media ${ }^{14,17,18}$.

Here we report a fundamentally different approach to dynamic control of the wavefront of light with light. It is based on coherent light-matter interaction ${ }^{19-23}$ on a static metasurface and offers radical advantages. We exploit that manifestations of optical phenomena in traveling and standing waves are different: a thin film or metasurface may be placed at an electric field node or antinode of the standing wave formed by counter-propagating coherent light beams, resulting in suppression or enhancement of the electric light-matter interaction, respectively (Figure 1). This approach has been shown to allow modulation of intensity ${ }^{24}$, propagation direction ${ }^{25}$ and polarization ${ }^{26}$ of light with high contrast, terahertz $(\mathrm{THz})$ bandwidth $^{27,28}$ and arbitrarily-low intensity levels down to single photons ${ }^{29}$. The only competitive all-optical solution for dynamic wavefront control is photorefraction $^{30-32}$, which relies on microsecond- to second-scale nonlinear interaction of light (which may be incoherent) and matter in a volume medium, making it very different from the femtosecondscale linear interaction of two coherent light beams and a planar medium reported here.

In fact what we are presenting here is an elementary version of an 'optical computer' that has the main characteristics of a Turing machine. It takes data (signal image) from one optical input and processes it using instructions (rules) taken from the second optical input (control image). The metasurface acts as the 'processor' that performs elementary logical functions. Our optical data processor operates in an analog, rather than a discrete, 'digital' fashion. Moreover, our computer operates in the parallel regime handling the entire image at once.

\section{MATERIALS AND METHODS}

Metasurface nanofabrication

2D coherent control of absorption of light with light is demonstrated using a metamaterial nanostructure fabricated starting with a $50-\mathrm{nm}$ thick low-stress silicon nitride membrane, which was $500 \times 500 \mu \mathrm{m}^{2}$ in size and supported by a silicon frame. A 60 -nm-thick gold layer was thermally evaporated onto the membrane and then the silicon nitride layer was removed by reactive ion etching (Plasmalab 80 Plus by Oxford Instruments) leaving a gold membrane. The free-standing gold

${ }^{1}$ Optoelectronics Research Centre and Centre for Photonic Metamaterials, University of Southampton, Highfield, Southampton SO17 $1 \mathrm{BJ}$, UK; ${ }^{2}$ Institute for Life Sciences, University of Southampton, Highfield, Southampton SO17 1BJ, UK and ${ }^{3}$ The Photonics Institute and Centre for Disruptive Photonic Technologies, Nanyang Technological University, Singapore 637371, Singapore

Correspondence: M Papaioannou, Email: mcp1g13@soton.ac.uk; E Plum, Email: erp@orc.soton.ac.uk; NI Zheludev, Email: niz@orc.soton.ac.uk

Received 15 September 2015; revised 7 January 2016; accepted 11 January 2016; accepted article preview online 14 January 2016 
film was structured by milling an array of split-ring apertures (Figure 2c) that has a $350 \times 350 \mathrm{~nm}^{2}$ unit cell using a focused ion beam system (Helios 600 NanoLab by FEI). The overall size of the metamaterial array is $100 \times 100 \mu \mathrm{m}^{2}$.

\section{Experimental characterization}

Coherent control of metasurface excitation with spatial resolution is achieved by projecting images onto the front and back of the freestanding gold metasurface using coherent light. The experimental setup is shown in Figure 2 and we provide relevant additional information here. Incident light was linearly polarized with the electric field parallel to the symmetry axis of the split-ring pattern throughout all experiments. The metasurface's transmission, reflection and absorption characteristics shown in Figure $2 \mathrm{~b}$ were measured using a microspectrophotometer (by CRAIC Technologies) equipped with an incoherent halogen light source. All other experiments were conducted with coherent light from a 785-nm continuous wave diode

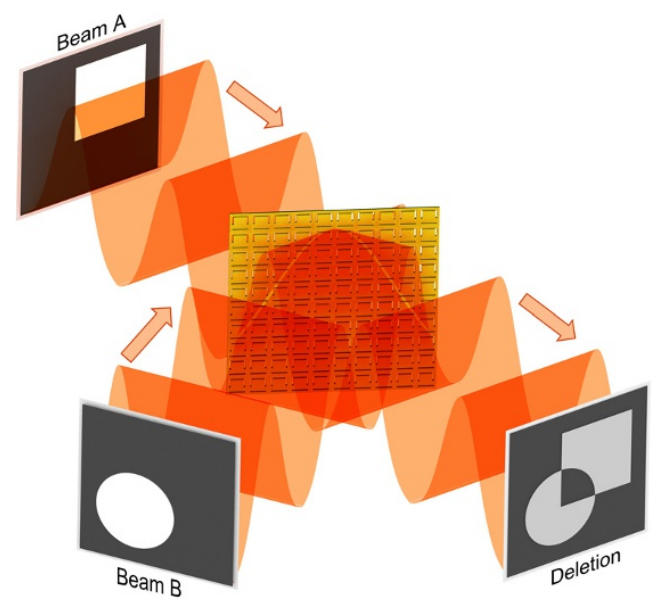

Figure 1 Two-dimensional control of absorption of light with light. Images A and $\mathrm{B}$ projected by coherent light onto an absorbing metasurface result in deletion (shown) or enhancement of common image features, depending on the phase difference between the interacting beams. laser. The intensity masks that provide the spatial intensity modulation shown in Figure 3a and $3 \mathrm{~b}$ were made by milling a $1.5 \mathrm{~mm}$ hole through an aluminum plate, whereas the intensity masks for the International Year of Light logo shown in Figure 4 were made by photolithography from a chromium layer supported by a glass substrate. The intensity masks were then imaged by a lens with $400 \mathrm{~mm}$ focal length and a $50 \times$ objective onto either side of the metasurface with 100-fold demagnification. One side of the metasurface was imaged onto a charge-coupled device (CCD) camera (Evolve 512 by Photometrics) using a beam splitter and a $200-\mathrm{mm}$ focal length lens.

\section{RESULTS AND DISCUSSION}

We demonstrate all-optical wavefront control by projecting images onto opposite sides of the free-standing gold metasurface using coherent light. By modulating the relative phase of the two beams, we control the absorption of light, and hence the wavefront, with high spatial resolution. This is illustrated by performing 2D all-optical Boolean logical operations as well as image processing, see Figure 1.

Destructive interference of coherent counter-propagating electromagnetic waves of equal intensity will cancel the electric field interacting with a metasurface placed at a node of the standing wave. In contrast, constructive interference doubles the electric field interacting with a metasurface placed at an antinode in comparison to illumination with a single beam. Although absorption in metasurfaces illuminated by a single wave is limited to $50 \%$, the interaction of two coherent waves allows absorption to be controlled continuously from 0 to $100 \%$ by changing the position of the metasurface relative to the standing wave ${ }^{24}$.

Following Fang et $a l^{33}$, such a linear metasurface with two counterpropagating incident beams $E_{\mathrm{A}}(x, y)$ and $E_{\mathrm{B}}(x, y)$ and two output beams $E_{\mathrm{C}}(x, y)$ and $E_{\mathrm{D}}(x, y)$ of the same linear polarization (i.e., the same polarization eigenstate of the metasurface) may be described by:

$$
\left(\begin{array}{c}
E_{\mathrm{D}} \\
E_{\mathrm{C}}
\end{array}\right)=\left(\begin{array}{cc}
s & s+1 \\
s+1 & s
\end{array}\right)\left(\begin{array}{c}
E_{\mathrm{A}} \\
E_{\mathrm{B}}
\end{array}\right)
$$

As metasurfaces scatter fields equally in the forward and backward directions, the structure is described by a single wavelength-dependent complex amplitude scattering parameter $s=s(\lambda)$ for the illuminating

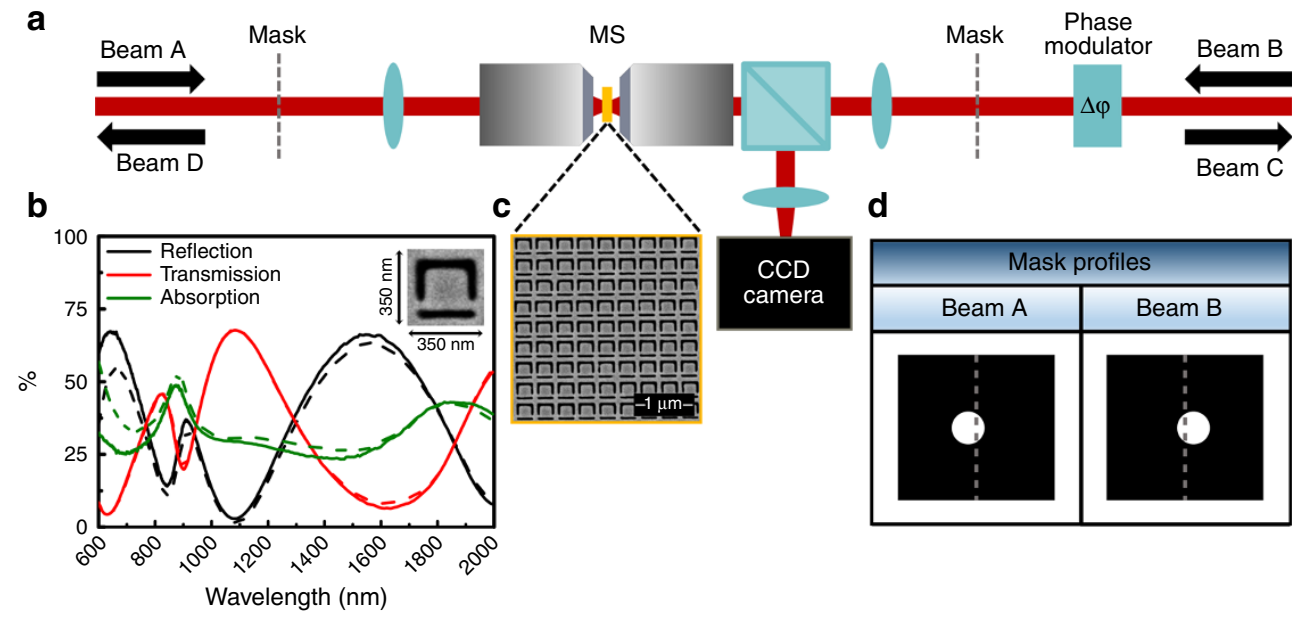

Figure 2 Two-dimensional all-optical control of light. (a) Schematic of the experimental setup where two-dimensional spatial modulation across coherent beams $A$ and $B$ is provided by masks and temporal modulation is provided by a phase modulator. A CCD camera monitors the system output. (b) Reflection, transmission and absorption spectra of the free-standing gold metasurface (MS) for illumination of its front (solid) and back (dashed). (c) Scanning electron microscopy image of a fragment of the MS. (d) Intensity mask designs imaged onto the MS to demonstrate coherent logical operations. 

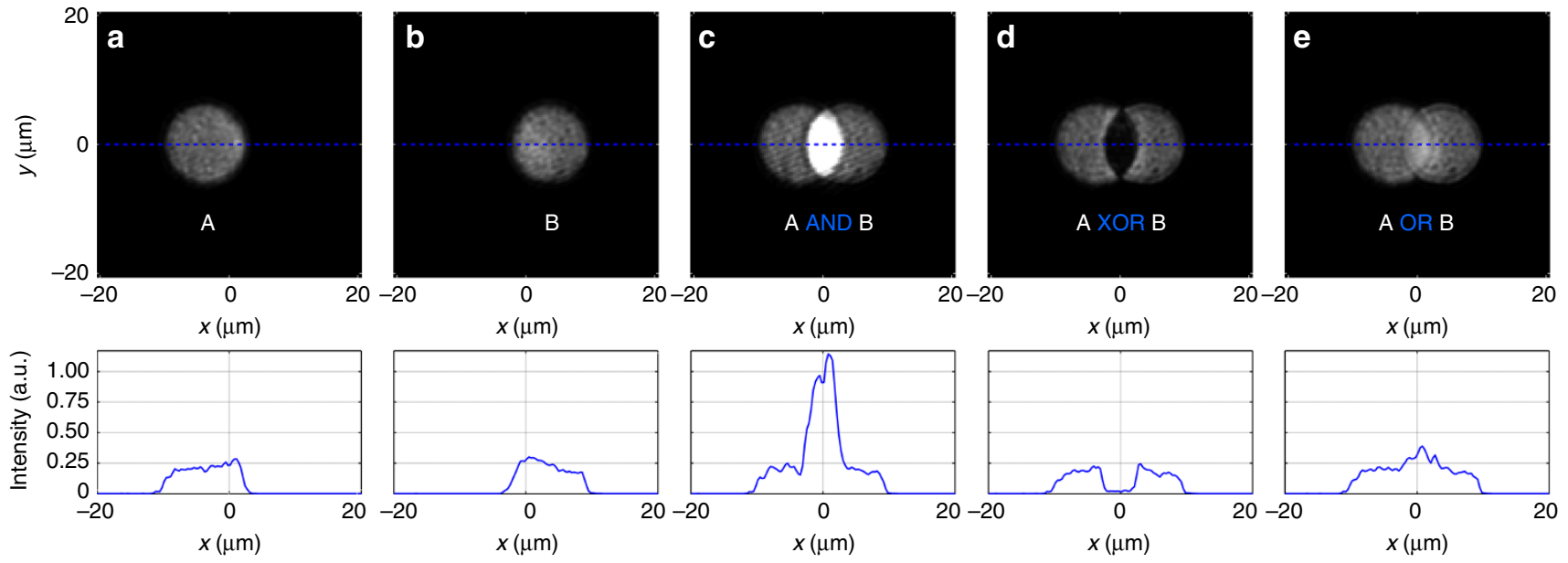

Figure 3 Two-dimensional coherent logical operations. Images of the metasurface illuminated by (a) beam A only, (b) beam B only and (c-e) both beams A and B. Different relative phases of beams A and B correspond to different logical operations: (c) A AND B, (d) A XOR B and (e) A OR B. Graphs show the intensity cross-section along the corresponding dashed blue line. Intensity levels are shown on the same grayscale for all images and on the same vertical scale for all graphs.
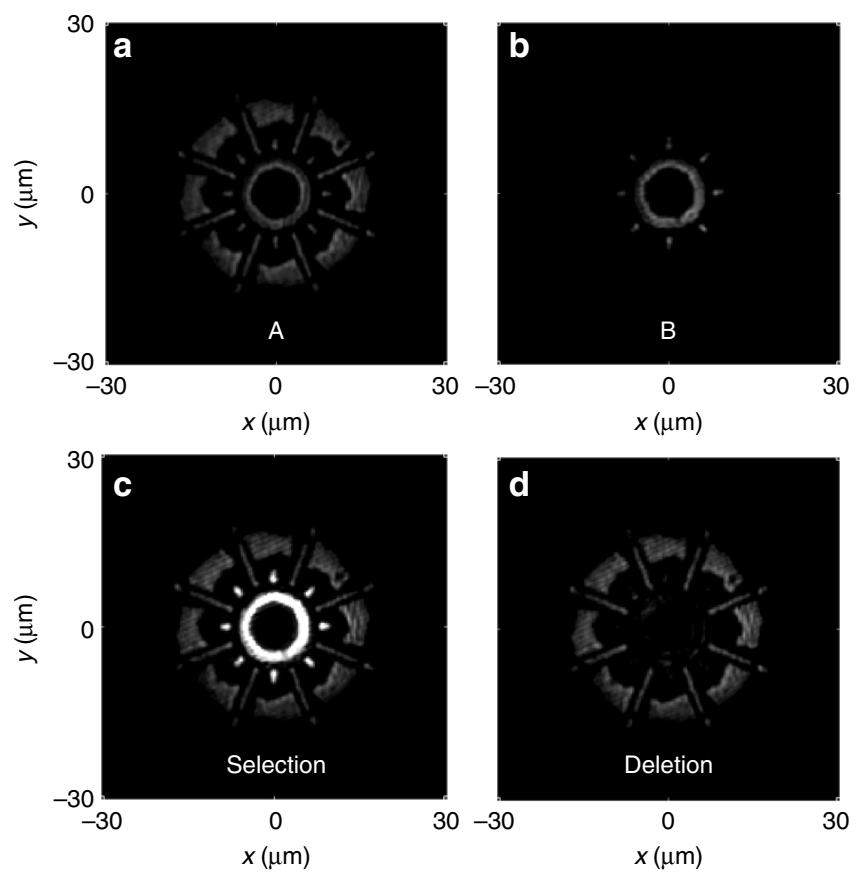

Figure 4 Coherent image processing. Images of the metasurface illuminated by (a) the International Year of Light logo (beam A) and (b) its central sun (beam B). Coherent (c) selection and (d) deletion of the sun within the logo for different relative phases of beams $A$ and $B$.

polarization eigenstate. Equation (1) describes each output beam (say $E_{\mathrm{C}}$ ) as the linear superposition of transmission and reflection of the beams illuminating the opposite $\left(E_{\mathrm{A}}\right)$ and same $\left(E_{\mathrm{B}}\right)$ side of the metasurface, respectively. Here, reflection of a single incident wave (say $E_{\mathrm{A}}$ ) corresponds to the scattered field $\left(s E_{\mathrm{A}}\right)$, and transmission corresponds to the superposition of scattered and incident fields $\left((s+1) E_{\mathrm{A}}\right)$.

The linearity of this system implies that equal scaling of all input intensities will result in scaling of all output intensities by the same factor. However, linearity does not imply a linear relationship between the output intensities and phase or intensity changes of only one of the input beams. Such changes are, in general, nonlinear and it has been shown theoretically that this enables the effectively nonlinear functions of all-optical transistors, summators and invertors ${ }^{33}$.

Coherent perfect absorption and perfect transparency, as described above, will be achieved for incident waves of equal intensity, $E_{\mathrm{A}}=e^{i \theta}$ and $E_{\mathrm{B}}=1$, illuminating a metasurface that absorbs $50 \%$ of a single illuminating beam described by $s=-0.5$, where $\theta=\theta(t)$ is the timedependent phase difference of the incident beams at the metasurface. Both output beams vanish for $\theta=0$ (perfect absorption, $E_{\mathrm{C}}=E_{\mathrm{D}}=0$ ), whereas $\theta=\pi$ corresponds to perfect transparency $\left(E_{\mathrm{C}}=E_{\mathrm{A}}\right.$ and $\left.E_{\mathrm{D}}=E_{\mathrm{B}}\right)$ and intermediate phases $\theta= \pm \pi / 3$ lead to $\left|E_{\mathrm{C}}\right|=\left|E_{\mathrm{D}}\right|=0.5$. The latter is the same as for illumination of the metasurface by only one beam, i.e., each output beam has $25 \%$ of the intensity (defined as $\left.I=|E|^{2}\right)$ of one illuminating beam.

Consequently, the output intensities depend on whether beams A and $\mathrm{B}$ are switched on or off, for a given phase $\theta$, which enables alloptical Boolean logical operations. For $\theta=\pi$, this corresponds to the truth table of the logical operation A AND B, with an intensity threshold between 0.25 and 1 :

\begin{tabular}{lll}
\hline $\boldsymbol{I}_{\mathbf{A}}$ & $\boldsymbol{I}_{\mathbf{B}}$ & $\boldsymbol{I}_{\mathbf{C}}=\boldsymbol{I}_{\mathbf{D}}$ \\
\hline 1 & 1 & 1 \\
1 & 0 & 0.25 \\
0 & 1 & 0.25 \\
0 & 0 & 0 \\
\hline
\end{tabular}

Similarly, $\theta=0$ corresponds to the truth table of A XOR B (or NOT A if $I_{\mathrm{B}}=1$ is fixed) with an intensity threshold between 0 and 0.25 :

\begin{tabular}{lll}
\hline$I_{A}$ & $I_{B}$ & $I_{C}=I_{D}$ \\
\hline 1 & 1 & 0 \\
1 & 0 & 0.25 \\
0 & 1 & 0.25 \\
0 & 0 & 0 \\
\hline
\end{tabular}


And finally, $\theta= \pm \pi / 3$ corresponds to A OR B with an intensity threshold between 0 and 0.25 :

\begin{tabular}{lll}
\hline $\boldsymbol{I}_{\mathrm{A}}$ & $\boldsymbol{I}_{\mathrm{B}}$ & $\boldsymbol{I}_{\mathrm{C}}=\boldsymbol{I}_{\mathbf{D}}$ \\
\hline 1 & 1 & 0.25 \\
1 & 0 & 0.25 \\
0 & 1 & 0.25 \\
0 & 0 & 0 \\
\hline
\end{tabular}

Ideal performance requires a thin film or metasurface of deeply subwavelength thickness that absorbs $50 \%$ of a single beam illuminating the structure. Exploiting that the nanostructure of metasurfaces can be carefully engineered to achieve suitable optical properties and, guided by numerical modeling, we fabricated a 60 -nm-thick free-standing gold metasurface with a split ring aperture unit cell and an absorption resonance near our experimental wavelength of $785 \mathrm{~nm}$, see Materials and Methods section as well as Figure 2. The free-standing gold nanostructure has almost identical optical properties for illumination of its front and back, where a small asymmetry results from different surface roughness on opposite sides, and it absorbs about $33 \%$ of a single illuminating beam (Figure 2b). To coherently control the metasurface excitation, we split the output of a 785-nm continuouswave laser along two paths of matched lengths, $\mathrm{A}$ and $\mathrm{B}$, that illuminate opposite sides of the nanostructure. Intensity masks are placed in both paths and imaged onto the metasurface to control the spatial intensity distribution on the metasurface (Figure 2a). In order to introduce temporal control, a liquid-crystal phase modulator is used to control the relative phase of the illuminating beams, which shifts the standing wave relative to the metasurface. This way, any part of the structure can be illuminated by a single beam (A or B) or by both coherent waves, where the phase modulator allows tuning from constructive to destructive interference at the metasurface position; that is from enhanced to negligible light-matter interaction. We detect the resulting coherent effects by imaging the metasurface onto a CCD camera, detecting output beam $\mathrm{C}$ that is formed by transmission of beam $\mathrm{A}$ and reflection of beam $\mathrm{B}$. We note that less-than-ideal absorption in our sample reduces the achievable level of coherent absorption and results in a difference between intensity levels in the output beams ${ }^{26}$. The small difference between experimental transmission and reflection levels is compensated for by adjusting the input intensities.

Figure 3 illustrates 2D control of light with light on the free-standing gold metasurface for the simplest possible case of two partially overlapping illuminating beams, A and B (panels (a) and (b)), realized by imaging the intensity profiles imposed by two metallic masks with slightly misaligned circular holes onto opposite sides of the metasurface. In areas of overlapping illuminating beams, the detected intensity depends dramatically on the relative phase difference between the incident waves. Noting that the intensities of incoherent beams would simply add up, we find that illumination with a second coherent beam leads to a range of output intensities from fourfold increase to almost complete suppression. The coherently enhanced output intensity (panel (c)) corresponds to a reduced light-matter interaction and thus reduced absorption near the standing wave's electric node. On the other hand, changing the phase of one illuminating beam by $\pi$ translates the standing wave, placing the metasurface near an electric antinode, where almost complete suppression of the detected output intensity occurs as a result of enhanced light-matter interaction and therefore enhanced absorption (panel (d)). Intermediate phases lead to the full range of intermediate intensities (panel (e)).
As discussed above, the coherently controlled interaction of the partially overlapping beams $A$ and $B$ on the metasurface corresponds to logical operations A AND B, A XOR B and A OR B, and the detected images strongly resemble the associated Venn diagrams of set theory, see Figure 3. Thus, coherent control provides an all-optical way of performing logical operations, which may be applied to arbitrary combinations of images. This is illustrated by Figure 4 for the logo of the International Year of Light (beam A, panel (a)) and the sun that forms its center (beam $\mathrm{B}$, panel (b)). In this case, reduced absorption near an electric field node of the standing wave results in fourfold enhancement or selection (recognition) of the logo's central sun, which both images have in common (panel (c)). On the other hand, a $\pi$ phase shift of one illuminating beam shifts the standing wave, placing the metasurface near an electric antinode, where the sun is strongly absorbed and therefore deleted from the logo, leaving just the flags that are present in only one image (panel (d)). In this way, 2D coherent control of light with light provides an all-optical method for image processing.

\section{CONCLUSIONS}

In the demonstrations described above, we exploited the interaction of coherent waves with spatially modulated wavefronts to control the light-matter interaction on a lossy metasurface in order to perform elementary all-optical computations. Constructive interference at the metasurface led to enhanced light-matter interaction and therefore absorption, whereas destructive interference suppressed the interaction of light with absorbing matter. 2D coherent control provides a practical solution for dynamic control over the expression of many properties of metasurfaces and thin films of sub-wavelength thickness, e.g., to control intensity, propagation direction ${ }^{25}$ and polarization ${ }^{26}$ of electromagnetic waves throughout the electromagnetic spectrum.

The spatial resolution of $2 \mathrm{D}$ coherent control of light with light is limited by the size of the meta-molecules, and the diffraction limit of the imaging system used to illuminate the metasurface. The metamolecules of non-diffracting metasurfaces are of sub-wavelength size and the diffraction limit is about one wavelength, therefore the achievable spatial resolution corresponds approximately to the wavelength of the illuminating wave. The temporal resolution is determined by the bandwidth of the metasurface's absorption resonance, leading to femtosecond-scale response times suitable for $\mathrm{THz}$ modulation frequencies in case of plasmonic resonances that have a typical quality factor on the order of 10, see Fang et al ${ }^{27}$. Recently, $100 \mathrm{THz}$ coherent optical switching has been demonstrated with plane waves ${ }^{28}$. Non-resonant and highly absorbing metallic thin films have been reported as a broadband alternative to metasurfaces at microwave frequencies ${ }^{34}$ and predicted to yield even higher bandwidth at optical frequencies ${ }^{35}$. The all-optical nature of the technique means it needs only a static metasurface (or thin film) and makes it applicable to many metasurface designs, including spatially varying one ${ }^{5-13,25}$. Furthermore, coherent control offers high contrast and it is compatible with arbitrarily low intensities down to the single-photon quantum regime ${ }^{29}$.

With femtosecond-scale response times, wavelength-scale spatial resolution, large optical contrast and quantum-level energy requirements, 2D control of light with light has potential applications ranging from tuneable, reconfigurable and adaptive optical devices, as well as coherent image and video processing to massively parallel modulation and processing of optical data and all-optical computing ${ }^{33}$. Our approach also allows selective amplification or deletion of modes from multimode signals and thus promises solutions for mode selection in spatial-mode multiplexing for higher bandwidth optical telecommunications. 


\section{CONFLICT OF INTEREST}

The authors declare no conflict of interest.

\section{ACKNOWLEDGEMENTS}

This work is supported by the UK's Defence Science and Technology Laboratory (Grant DSTLX1000064081), the MOE Singapore (Grant MOE2011-T3-1-005), the Leverhulme Trust, the University of Southampton Enterprise Fund and the UK's Engineering and Physical Sciences Research Council (Grant EP/G060363/1). The data from this paper can be obtained from the University of Southampton ePrints research repository: http://dx.doi.org/10.5258/SOTON/379907.

1 Leonhardt U. Optical conformal mapping. Science 2006; 312: 1777-1780.

2 Schurig D, Mock JJ, Justice BJ, Cummer SA, Pendry JB et al. Metamaterial electromagnetic cloak at microwave frequencies. Science 2006; 314: 977-980.

3 Cai WS, Chettiar UK, Kildishev AV, Shalaev VM. Optical cloaking with metamaterials. Nat Photonics 2007; 1: 224-227.

4 Valentine J, Li J, Zentgraf T, Bartal G, Zhang X. An optical cloak made of dielectrics. Nat Mater 2009; 8: 568-571.

5 Yu NF, Genevet P, Kats MA, Aieta F, Tetienne J-P et al. Light propagation with phase discontinuities: generalized laws of reflection and refraction. Science 2011; 334: 333-337.

6 Papakostas A, Potts A, Bagnall DM, Prosvirnin SL, Coles HJ et al. Optical manifestations of planar chirality. Phys Rev Lett 2003; 90: 107404

7 Sun SL, Yang KY, Wang CM, Juan TK, Chen WT et al. High-efficiency broadband anomalous reflection by gradient meta-surfaces. Nano Lett 2012; 12: 6223-6229.

8 Walther B, Helgert C, Rockstuhl C, Setzpfandt F, Eilenberger F et al. Spatial and spectral light shaping with metamaterials. Adv Mater 2012; 24: 6300-6304.

9 Roy T, Nikolaenko AE, Rogers ETF. A meta-diffraction-grating for visible light. J Opt 2013; 15: 085101.

$10 \mathrm{Ni}$ XJ, Ishii S, Kildishev AV, Shalaev VM. Ultra-thin, planar, Babinet-inverted plasmonic metalenses. Light Sci App/ 2013; 2: e72, doi:10.1038/lsa.2013.28.

11 Lin DM, Fan PY, Hasman E, Brongersma ML. Dielectric gradient metasurface optical elements. Science 2014; 345: 298-302.

12 Chen WT, Yang KY, Wang CM, Huang YW, Sun G et al. High-efficiency broadband metahologram with polarization-controlled dual images. Nano Lett 2014; 14: 225-230.

13 Veksler D, Maguid E, Shitrit N, Ozeri D, Kleiner V et al. Multiple wavefront shaping by metasurface based on mixed random antenna groups. ACS Photonics 2015; 2: 661-667.

14 Efron U. Spatial Light Modulator Technology: Materials, Devices, and Applications. New York: CRC Press; 1994.

15 Lazarev G, Hermerschmidt A, Krüger S, Osten S. LCOS Spatial Light Modulators: Trends and Applications. Weinheim: Wiley-VCH Verlag GmbH \& Co. KGaA; 2012, 1-29.

$16 \mathrm{Yu} \mathrm{H}$, Park J, Lee K, Yoon J, Kim K et al. Recent advances in wavefront shaping techniques for biomedical applications. Curr Appl Phys 2015; 15: 632-641.

17 Günter P. Holography, coherent light amplification and optical phase conjugation with photorefractive materials. Phys Rep 1982; 93: 199-299.
18 Xu X, Liu HL, Wang LV. Time-reversed ultrasonically encoded optical focusing into scattering media. Nat Photonics 2011; 5: 154-157.

19 Stockman MI, Faleev SV, Bergman DJ. Coherent control of femtosecond energy localization in nanosystems. Phys Rev Lett 2002; 88: 067402.

20 Gjonaj B, Aulbach J, Johnson P, Mosk A, Kuipers L et al. Active spatial control of plasmonic fields. Nat Photonics 2011; 5: 360-363.

21 Wan WJ, Chong YD, Ge L, Noh H, Stone AD et al. Time-reversed lasing and interferometric control of absorption. Science 2011; 331: 889-892.

22 Li ZP, Zhang SP, Halas NJ, Nordlander P, Xu HX. Coherent modulation of propagating plasmons in silver-nanowire-based structures. Small 2011; 7: 593-596.

23 Yoon JW, Koh GM, Song SH, Magnusson R. Measurement and modeling of a complete optical absorption and scattering by coherent surface plasmon-polariton excitation using a silver thin-film grating. Phys Rev Lett 2012; 109: 257402.

24 Zhang J, MacDonald KF, Zheludev NI. Controlling light-with-light without nonlinearity. Light Sci App/ 2012; 1: e18, doi:10.1038/lsa.2012.18.

25 Shi J, Fang X, Rogers ETF, Plum E, MacDonald KF et al. Coherent control of Snell's Law at metasurfaces. Opt Express 2014; 22: 21051-21060.

26 Mousavi SA, Plum E, Shi J, Zheludev NI. Coherent control of optical polarization effects in metamaterials. Sci Rep 2015; 5: 8977.

27 Fang X, Tseng ML, Ou JY, MacDonald KF, Tsai DP et al. Ultrafast all-optical switching via coherent modulation of metamaterial absorption. App/ Phys Lett 2014; 104: 141102.

28 Nalla V, Vezzoli S, Valente J, Handong S, Zheludev NI. $100 \mathrm{THz}$ optical switching with plasmonic metamaterial. CLEO/Europe-EQEC, Munich, Germany, 21-25 June 2015

29 Roger T, Vezzoli S, Bolduc E, Valente J, Heitz JJF et al. Coherent perfect absorption in deeply subwavelength films in the single-photon regime. Nat Commun 2015; 6: 7031.

30 Klein MB. Beam coupling in undoped GaAs at $1.06 \mu \mathrm{m}$ using the photorefractive effect. Opt Lett 1984; 9: 350-352.

31 Voit $\mathrm{E}$, Zha $\mathrm{MZ}$, Amrhein $\mathrm{P}$, Günter $\mathrm{P}$. Reduced $\mathrm{KNbO}_{3}$ crystals for fast photorefractive nonlinear optics. App/ Phys Lett 1987; 51: 2079-2081.

32 Herlocker JA, Ferrio KB, Hendrickx E, Guenther BD, Mery S et al. Direct observation of orientation limit in a fast photorefractive polymer composite. Appl Phys Lett 1999; 74: 2253-2255.

33 Fang X, MacDonald KF, Zheludev NI. Controlling light with light using coherent metadevices: all-optical transistor, summator and invertor. Light Sci Appl 2015; 4: e292, doi:10.1038/lsa.2015.65.

34 Li SC, Luo J, Anwar S, Li S, Lu WX et al. Broadband perfect absorption of ultrathin conductive films with coherent illumination: superabsorption of microwave radiation. Phys Rev B 2015; 91: 220301(R).

35 Pu MB, Feng Q, Wang M, Hu CG, Huang C et al. Ultrathin broadband nearly perfect absorber with symmetrical coherent illumination. Opt Express 2012; 20: 2246-2254.

\footnotetext{
(c) (1) $\odot$ This work is licensed under a Creative Commons Attributionc. NonCommercial-NoDerivs 4.0 International License. The images or other third party material in this article are included in the article's Creative Commons license, unless indicated otherwise in the credit line; if the material is not included under the Creative Commons license, users will need to obtain permission from the license holder to reproduce the material. To view a copy of this license, visit http:// creativecommons.org/licenses/by-nc-nd/4.0/
} 